\title{
An optimized isolation and labeling platform for accurate microRNA expression profiling
}

\author{
JACLYN SHINGARA, ${ }^{1}$ KERRI KEIGER, ${ }^{1}$ JEFFREY SHELTON, WALAIRAT LAOSINCHAI-WOLF, \\ PATRICIA POWERS, RICHARD CONRAD, DAVID BROWN, and EMMANUEL LABOURIER \\ Ambion, Inc., Austin, Texas 78744-1832, USA
}

\begin{abstract}
MicroRNAs (miRNAs) are small, noncoding RNAs that regulate gene expression in both plants and animals. miRNA genes have been implicated in a variety of important biological processes, including development, differentiation, apoptosis, fat metabolism, viral infection, and cancer. Similar to protein-coding messenger RNAs, miRNA expression varies between tissues and developmental states. To acquire a better understanding of global miRNA expression in tissues and cells, we have developed isolation, labeling, and array procedures to measure the relative abundance of all of the known human mature miRNAs. The method relies on rapid isolation of RNA species smaller than $\sim \mathbf{4 0}$ nucleotides (nt), direct and homogenous enzymatic labeling of the mature miRNAs with amine modified ribonucleotides, and hybridization to antisense DNA oligonucleotide probes. A thorough performance study showed that this miRNA microarray system can detect subfemtomole amounts of individual miRNAs from $<1 \mu \mathrm{g}$ of total RNA, with $\mathbf{9 8 \%}$ correlation between independent replicates. The system has been applied to compare the global miRNA expression profiles in 26 different normal human tissues. This comprehensive analysis identified miRNAs that are preferentially expressed in one or a few related tissues and revealed that human adult tissues have unique miRNA profiles. This implicates miRNAs as important components of tissue development and differentiation. Taken together, these results emphasize the immense potential of microarrays for sensitive and high-throughput analysis of miRNA expression in normal and disease states.
\end{abstract}

Keywords: microRNA; miRNA; gene expression profile; oligonucleotide microarray; poly(A) polymerase

\section{INTRODUCTION}

MicroRNAs (miRNAs) are small ( $\sim 22$-nucleotide) RNAs that are encoded in the genomes of animals, plants, and viruses (Ambros 2004; Bartel and Chen 2004; Pfeffer et al. 2004). The short, single-stranded RNAs are generated from longer primary miRNA transcripts (pri-miRNAs) by a stepwise process (Lee et al. 2002; Murchison and Hannon 2004). The first step in miRNA biogenesis takes place in a multiprotein nuclear complex, termed the Microprocessor (Denli et al. 2004; Gregory et al. 2004). There, pri-miRNAs are cleaved by an RNase III family nuclease, Drosha, to generate precursor miRNAs (pre-miRNAs) (Lee et al. 2003; Zeng et al. 2004). The pre-miRNAs, which are predicted to form hairpins of 70-125 nt with a 2-nt $3^{\prime}$ overhang, are exported to the cytoplasm by a RanGTP/exportin 5-depen-

\footnotetext{
${ }^{1}$ These authors contributed equally to this work.

Reprint request to: Emmanuel Labourier, Ambion, Inc., 2130 Woodward Street, Austin, TX 78744-1832, USA; e-mail: elabourier@ambion. com; fax: (512) 651-0201.

Article published online ahead of print. Article and publication date are at http://www.rnajournal.org/cgi/doi/10.1261/rna.2610405.
}

dent mechanism (Yi et al. 2003; Bohnsack et al. 2004; Lund et al. 2004; Zeng and Cullen 2004). In the cytoplasm, premiRNAs are recognized and processed by a second RNase III-family enzyme called Dicer to generate $~ 22-b p$ RNA duplexes with 2-nt $3^{\prime}$ overhangs (Grishok et al. 2001; Hutvagner et al. 2001; Ketting et al. 2001; Zhang et al. 2004). Mature miRNAs enter RISC (RNA-induced silencing complex), where one strand of the RNA duplex will guide RISC to induce endonucleolytic cleavage or translational repression of specific target messenger RNAs (Olsen and Ambros 1999; Llave et al. 2002; Zeng et al. 2002; Doench et al. 2003; Schwarz et al. 2003; Yekta et al. 2004).

Biochemical and bioinformatics approaches have identified several hundred genes encoding miRNAs in plants, Caenorhabditis elegans, Drosophila, and mammals. As with protein-coding genes, a key to understanding how miRNAs are functioning is to determine when and where they are expressed. Northern blots are commonly used for miRNA analysis, and interesting developmental or tissue-specific miRNA expression patterns have been identified (LagosQuintana et al. 2002; Houbaviy et al. 2003; Lim et al. 2003; Sempere et al. 2004). A variety of improvements or 
adaptation to existing technologies have also been tailored to small RNA detection. These include locked nucleic-acidmodified probes for Northern blot (Valoczi et al. 2004), oligonucleotide filter macroarrays (Krichevsky et al. 2003; Sioud and Rosok 2004), RNA oligonucleotide ligation followed by RT-PCR amplification (Grad et al. 2003), fluorescence resonance energy transfer (Allawi et al. 2004), signal-amplifying ribozymes (Hartig et al. 2004), primer extension (Zeng and Cullen 2003), nuclease protection (Overhoff et al. 2004), or direct real-time PCR amplification for detection of precursor miRNAs (Schmittgen et al. 2004). In addition, a number of microarray-based methods have recently been developed (Babak et al. 2004; Barad et al. 2004; Liu et al. 2004; Miska et al. 2004; Nelson et al. 2004; Sun et al. 2004; Thomson et al. 2004; Baskerville and Bartel 2005).

Conceptually, microarrays offer an ideal method for highthroughput, multiplex miRNA gene expression analyses in tissues and cells. However, miRNAs present unique challenges that make them more difficult to analyze than mRNAs. The inherent small size of miRNAs provides very little sequence for appending label or for designing probes. Furthermore, miRNAs represent only a small fraction ( $\sim 0.01 \%)$ of the mass of a total RNA sample, making it important to label them with the highest specific activity possible. Finally, miRNAs exist in three forms, short mature miRNA, hairpin pre-miRNA, and long pri-miRNA (Lee et al. 2003, 2004; Cai et al. 2004; Rodriguez et al. 2004). Since only the mature miRNA is active, it is important to eliminate array signal from the pre-miRNAs and pri-miRNAs. These factors conspire to make it difficult to detect miRNAs using standard array procedures and are not completely addressed by the procedures that have thus far been developed for miRNA array analysis. To eliminate the potential bias associated with random labeling or priming (Babak et al. 2004; Liu et al. 2004; Sun et al. 2004), detection of pre-miRNA and pri-miRNA sequences (Babak et al. 2004; Liu et al. 2004; Nelson et al. 2004; Sun et al. 2004), and PCR-based amplification or ligation strategies (Barad et al. 2004; Miska et al. 2004; Thomson et al. 2004; Baskerville and Bartel 2005), we have developed a method allowing direct labeling and hybridization of the mature and active miRNAs. The microarray system was evaluated using both artificial and real samples to determine its sensitivity, dynamic range, accuracy, and reproducibility. Finally, we used the system to analyze 26 human tissue samples, which enabled classification of normal tissues based on their miRNA expression profiles.

\section{RESULTS}

\section{Microarray strategy, design, and optimization}

Labeling a total RNA sample or fractions enriched in small RNAs results in labeled mature and precursor miRNAs. Because very little is known about how mature and precursor
miRNAs accumulate in cells and tissues, we chose to purify the active, mature miRNAs from samples so that we could exclude any artifacts that might be introduced in our array experiments by including the inactive precursors of the miRNAs. Routinely, purification of nucleic acid species by size requires separating the samples on electrophoretic gels, followed by localization of the samples on the gel, excision of the region of interest, and elution of the nucleic acid from the gel pieces. To minimize variability, and the time and effort required to isolate miRNAs from a total RNA sample, we developed a gel electrophoresis device that can recover mature miRNAs in $<15$ min without the need to excise and extract miRNAs from the gel matrix (see Materials and Methods). Efficient and reproducible miRNA recovery was demonstrated with $5^{\prime}$ radiolabeled synthetic miRNA oligonucleotides spiked in total RNA samples, and by direct analysis of natural miRNAs isolated from different input amounts of total RNA (see Supplemental Fig. 1).

To maximize the label from each miRNA without jeopardizing its capacity to hybridize to probes on a planar array, we next developed a tailing/labeling procedure. After evaluation of different template-independent polymerases and optimization of various reaction parameters, such as buffer composition, incubation time, enzyme and nucleotide type, or relative reagents concentration, we found that polynucleotide tails 20-50 nt long can reproducibly be appended to the $3^{\prime}$ ends of all miRNAs by the poly(A) polymerase (PAP) enzyme (data not shown). The $3^{\prime}$ tail is a mixture of standard and amine-modified nucleotides, and tailed miRNAs can subsequently be labeled with any mono-reactive NHS-ester dyes, such as Cy3 and Cy5 (Fig. 1). Using an optimized set of reaction conditions and ratio of modified to unmodified nucleotides improve tailing efficiency and reproducibility and reduce the fluorescence quenching that occurs when dye molecules are located on adjacent or nearby nucleotides (data not shown).

To optimize the miRNA probe design, fluorescently labeled miRNAs from human thymus, lung, and brain were hybridized to series of immobilized oligonucleotide probes with regions complementary to eight different miRNAs and various $5^{\prime}$ and $3^{\prime}$ linker lengths and sequences connecting to the attachment moiety. A matrix of hybridization and washing time, temperature, and buffer compositions was used to identify the probe design and protocol that (1) maximized signal without creating significant signal from negative control elements spotted on the same microarrays and (2) provided relative miRNA abundance data congruent to Northern blot data (data not shown). This optimal probe design and array protocol (Fig. 1; see also Materials and Methods) was used for all subsequent studies.

\section{Microarray performances}

The overall performance of this new microarray system was evaluated by measuring several key parameters such as 


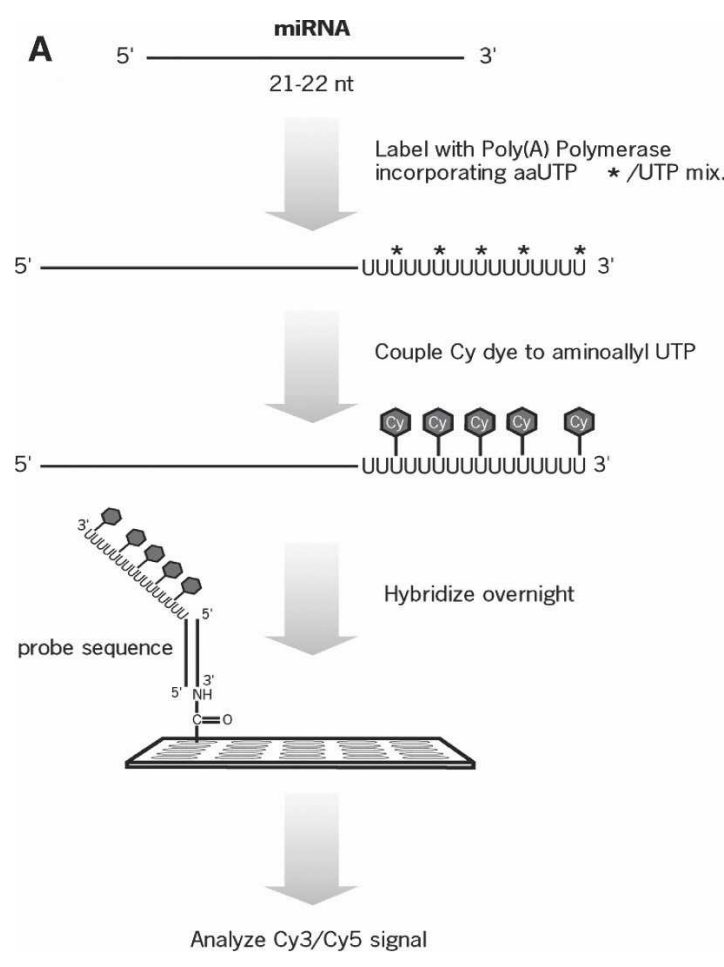

FIGURE 1. Overview of the miRNA microarray procedure. RNA species smaller than $\sim 40 \mathrm{nt}$ are purified by a rapid electrophoretic gel fractionation method. miRNAs are 3 '-end labeled with poly(A) polymerase, amine-modified nucleotides, and amine-reactive dyes. The fluorescently labeled miRNAs are hybridized to a glass slide on which miRNA-specific DNA oligonucleotide probes have been arrayed. Microarrays are processed and analyzed using standard equipment, scanner, and software. A representative result from a scanned microarray is shown in $B$.

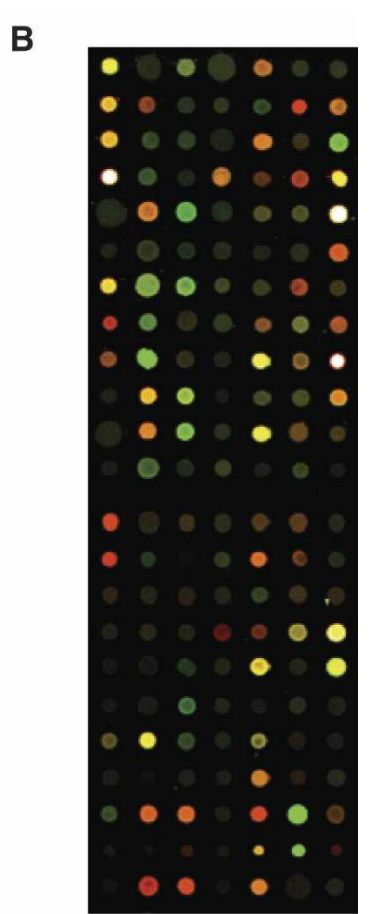

miRNA fraction purified from human liver (labeled with Cy5), and these samples were compared to the normal liver miRNA population (labeled with Cy3). Analysis of the mean intensities and the $\log _{2}$ (Normalized Ratio) of the miR-124 signal indicated that $0.42 \mathrm{fmol}$ of miR124 provides signal that is 5.8 -fold above the normal liver signal, and that even 0.14 fmol of miR-124 provides signal that can be clearly distinguished from the background signal (Table 1). This level of sensitivity allows detection of miRNAs that represent as little as $0.1 \%$ of the overall miRNA population in a $10-\mu \mathrm{g}$ total RNA sample.

To test the robustness and reproducibility of the system, miRNA expression profiles in human colon and prostate were compared in six independent array experiments. The $\log _{2}$ (Normalized Ratio) of each miRNA feature is shown in Figure 3A, and specific examples of miRNA overexpressed in prostate (let-7A, let-7C), overexpressed in colon (miR-200B) or with no significant variation (miR-16) are shown in Figure $3 \mathrm{~B}$. The average correlation between the six independent experiments was $98 \%$, with a standard deviation smaller than

consistency, accuracy/precision, sensitivity/limit of detection, and robustness/reproducibility. First, we compared two miRNA populations independently purified from the same tissue and labeled either with Cy5 or Cy3 (self vs. self analysis). Analysis of the mean signal in each channel showed equivalent labeling between both dyes $\left(R^{2}=0.992\right)$ and consistency of the intensities $(99.6 \%$ correlation) (Fig. 2A). Although microarrays are generally used to measure relative expression levels based on a given reference, we next wanted to determine how quantitative the miRNA microarray system was. Two artificial populations of 17 synthetic miRNAs mixed at different ratios were created, labeled with Cy5 or $\mathrm{Cy} 3$, and hybridized on the same array in triplicate (Fig. 2B). The $\log _{2}$ (Normalized Ratio) was measured for each miRNA feature and was used to calculate the relative representation of each miRNA in the two populations. The correlation between the theoretical and measured values was $84 \%$. The average difference between the theoretical and measured values was $3.5 \%$ with a maximum of $15 \%$, indicating that the microarray system can accurately report variations greater than $\sim 20 \%$.

We next determined the sensitivity of the array system by spiking various amounts of a synthetic miRNA into a sample that lacked this miRNA. Previous reports indicate that miR124 is specific to brain tissue (Lagos-Quintana et al. 2002). Thus, $0.14-140 \mathrm{fmol}$ of synthetic miR-124 were spiked in a
0.3 for individual miRNA, indicating that the miRNA microarray process is highly reproducible.

\section{Microarray validation}

As miRNAs represent only a minuscule fraction $(\sim 0.01 \%)$ of the mass of a total RNA sample, we next asked whether the differential miRNA expression levels reported by our microarray system precisely reflected the miRNA populations present in the starting total RNA samples. To test both the gel purification and the PAP labeling steps, we first isolated total RNA from human bladder, lung, or uterus and used these samples to determine miRNA expression levels either by microarray analysis after gel purification of the miRNA fraction (162 miRNAs), or directly in the total RNA samples by two independent methods: hybridization in solution (RNase protection assay, 3 miRNAs) and hybridization on solid support (Northern blot, 5 miRNAs). Analysis of the relative differential miRNA expression by tissue pairs showed a good correlation between the three independent detection methods (Fig. 4), indicating that the gel fractionation method uniformly captures miRNAs. Further, the experiment validates the accuracy of the array system in detecting both large (e.g., miR-21 and -143) and small variations of miRNA expression (e.g., miR-15A, -16, -20, -101, and -191). 
A

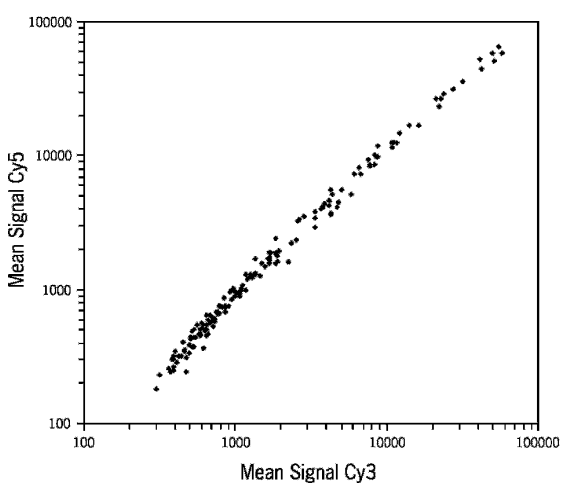

B

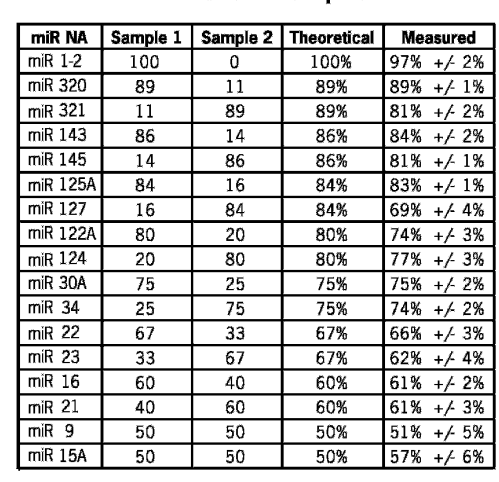

FIGURE 2. miRNA microarray accuracy and precision. (A) Self versus self analysis. A human thymus total RNA sample $(20 \mu \mathrm{g})$ was split in half and miRNAs were independently gel purified, labeled either with Cy3 or Cy5 dyes, and hybridized to the same array carrying 162 miRNA-specific probes. The graph shows the mean signal intensities at $532 \mathrm{~nm}$ (Cy3) and 635 $\mathrm{nm}$ (Cy5) for each miRNA probe. (B) Analysis of artificial samples. Chemically synthesized RNA oligonucleotides corresponding to 17 different mature miRNA sequences were mixed as indicated (amounts are in picograms) to create two artificial miRNA populations. Each sample was labeled with Cy 3 or Cy5 and hybridized to the same array. The measured percentage was calculated from the normalized $\mathrm{Cy} 3 / \mathrm{Cy} 5$ or $\mathrm{Cy} 5 / \mathrm{Cy} 3$ ratio from triplicate array analyses.

To determine whether the gel purification step provides any benefit, we compared the expression profiles obtained from gel-purified miRNA fractions and from fractions merely enriched in RNA smaller than $\sim 200 \mathrm{nt}$. The later fractions contain mostly small structural RNAs and transfer RNAs, and miRNAs represent only $\sim 0.1 \%$ of the sample mass. Both types of fraction were prepared from 10,5 , or $1 \mu \mathrm{g}$ of total RNA isolated from human bladder or lung. The miRNA fraction gel purified from $10 \mu \mathrm{g}$ total RNA, where the accuracy of miRNA expression levels was confirmed by independent detection methods (see Fig. 4; data not shown), was used as the reference sample. Hierarchical clustering analysis revealed that enriched fractions produced slightly different profiles and were less and less accurate as the sample input was reduced (Fig. 5A). These variations were accompanied by a strong reduction of the overall fluorescent signal on the array, even at the highest sample input (Fig. 5B). Further analysis showed a very poor correlation between the different inputs of enriched samples, and this loss of correlation was mainly attributed to the lowintensity signals (Fig. 5C, top). In contrast, gel-purified miRNAs from 5 and $1 \mu \mathrm{g}$ of total RNA resulted in $94 \%$ and $70 \%$ correlation relative to the reference $10-\mu \mathrm{g}$ sample for both high- and low-abundance miRNAs (Fig. 5C, bottom). Thus, miRNA labeling and profiling in a complex nucleic acid mixture is practical, but removing longer, unrelated RNA species by a gel purification process results in more accurate profiles and enables analysis at lower sample input.

\section{miRNA expression in human normal tissues}

Differential expression of specific miRNAs has been reported in several publications, suggesting a function for
miRNA in development and differentiation of adult tissues. To gain a better understanding of the global function of miRNA in adult tissue homeostasis, we analyzed the expression profiles of 162 known human miRNAs in 26 different tissues. The miRNA population from each tissue was compared to a reference sample consisting of a pool of each total RNA sample mixed in equal amount. After gel fractionation, labeling, and hybridization to custom glass slide arrays, the $\log _{2}$ (Normalized Ratio) was determined for each miRNA (see Supplemental Table 1), and the tissue and miRNA profiles were sorted based on miRNA expression levels across all tissues using hierarchical clustering (Fig. 6). As expected, individual miRNAs are present at higher (red), lower (green), or similar (black) levels in different tissues relative to the pool sample, which represents the average expression level of each miRNA in the global miRNA population from the 26 tissues.

Interestingly, there is a subset of miRNAs that are expressed at much higher levels in one or two tissues than they are in the rest of the tissues assayed. Among these are a handful that had been previously reported to be tissue specific (e.g., miR-1, -9, -122A, and -124) as well as more than 40 other miRNAs with a strong enrichment in restricted tissues (e.g., miR-99A, -134, -181A, and -205; see also Supplemental Table 1). In contrast, only a few miRNAs, such as miR-30A, $-33,-93$, or -140 , are expressed at roughly the same level in all adult tissues. Hierarchical clustering of the tissues based on their respective miRNA profiles also revealed that miRNA expression is similar between related tissues and distinct between unrelated tissues. For example, heart and skeletal muscle profiles are very similar, and the digestive tract tissues or the reproduc-

\begin{tabular}{lcccc}
\multicolumn{6}{l}{ TABLE 1. } \\
$\begin{array}{l}\text { fmol } \\
\text { miR-124 }\end{array}$ & $\begin{array}{c}\text { Mean Cy5 } \\
\text { signal }\end{array}$ & $\begin{array}{c}\text { Mean Cy5 } \\
\text { background }\end{array}$ & $\begin{array}{c}\text { Signal/ } \\
\text { background }\end{array}$ & $\begin{array}{c}\log _{2} \\
(\text { Cy5/Cy3) }\end{array}$ \\
\hline 0.14 & 213 & 55 & 3.87 & 1.90 \\
0.42 & 403 & 65 & 6.20 & 2.53 \\
1.41 & 796 & 53 & 15.02 & 3.61 \\
42.4 & 2877 & 73 & 39.41 & 3.98 \\
141 & 19245 & 55 & 349.91 & 7.94 \\
\hline
\end{tabular}

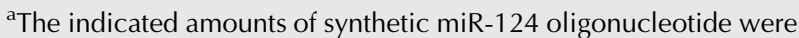
spiked in miRNA fractions isolated from $10 \mu \mathrm{g}$ of human liver total RNA and subjected to the miRNA microarray process.

b(Cy3) Human liver miRNAs. (Cy5) Human liver miRNAs plus spiked miR-124 oligonucleotide. 


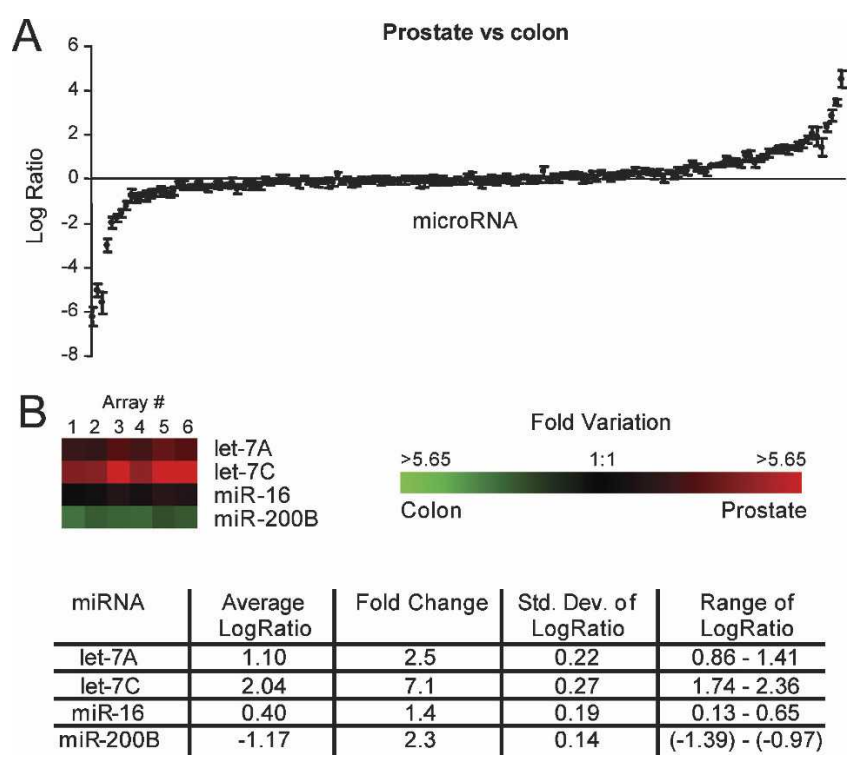

FIGURE 3. miRNA microarray reproducibility. Total RNA was isolated from a single human prostate sample and a single human colon sample. Each total RNA sample was split into six independent samples (10 $\mu \mathrm{g}$ each). miRNAs in each sample were independently fractionated and labeled. Each of the six Cy5-labeled prostate miRNA samples was mixed with a Cy3-labeled colon sample and the six prostate/colon pairs were combined and hybridized to six arrays. (A) Distribution of average LogRatio. LogRatio and standard deviation for each of the 162 miRNAspecific probes on the array are shown. The average standard deviation of LogRatio across replicates was 0.15 and the average correlation between the replicates was $98 \%$, ranging from $96.7 \%$ to $99.8 \%$. (B) Examples of LogRatio and corresponding absolute fold change for let7A, let-7C, miR-16, and miR-200B miRNAs. Red illustrates higher expression in prostate, while green depicts higher expression in colon. $\operatorname{LogRatio}=\log _{2}($ normalized ratio prostate/colon signal $)$.

tive organ tissues cluster together. Strikingly, the brain miRNA profile is clearly distinct from all the other tissues analyzed.

To validate the profiling data, new total RNA samples were isolated from 10 randomly selected tissues and miRNA expression levels were analyzed by independent detection methods. Both Northern blot (let-7C) and hybridization in solution (miR-200B) confirmed differential expression of individual miRNAs across tissues as well as differences in relative miRNA abundance within the same tissue (Fig. 7). miR-200B could not be detected in adrenal gland, skeletal muscle, and spleen. Tissues such as kidney, prostate, or thymus all have high expression levels of let-7C and miR200B, while liver poorly expresses these two miRNAs. Colon has higher expression levels of miR-200B but lower levels of let-7C relative to prostate. The same result was obtained by direct comparison between these two tissues (see Fig. 3B). Differential miRNA expression across tissues was also confirmed by pair analysis of bladder, lung, and uterus samples (see Fig. 4) or by testing other miRNAs, either broadly expressed across tissues (e.g., miR-16 and -22) or tissue specific (e.g., miR-124) (see Supplemental
Fig. 2). Collectively, these data show that the miRNA microarray system described here is a powerful and robust tool for high-throughput study of miRNA expression levels and comparative analysis of miRNA expression profiles.

\section{DISCUSSION}

Given their apparent importance in regulating gene expression, miRNAs offer a very interesting bio-molecule for life science research. A key to understanding how miRNAs are functioning in organisms is to know where and when they are expressed. To facilitate miRNA expression studies, we have adapted microarray instrumentation and protocols to analyze all of the known human miRNAs. The procedure incorporates miRNA purification, miRNA labeling through a poly-nucleotide tail appended to each miRNA, hybridization of the labeled miRNAs to a glass microarray, and microarray analysis using standard scanners and available software. The miRNA microarray system can detect subfemtomole amounts of miRNA, making it possible to detect even relatively rare miRNAs. The system provides high reproducibility ( $98 \%$ average correlation) and is reasonably quantitative, providing a great deal of confidence in the array results. Both Northern blot analysis and solution hybridization methods were used to validate relative miRNA expression levels within the same sample and across human tissues.

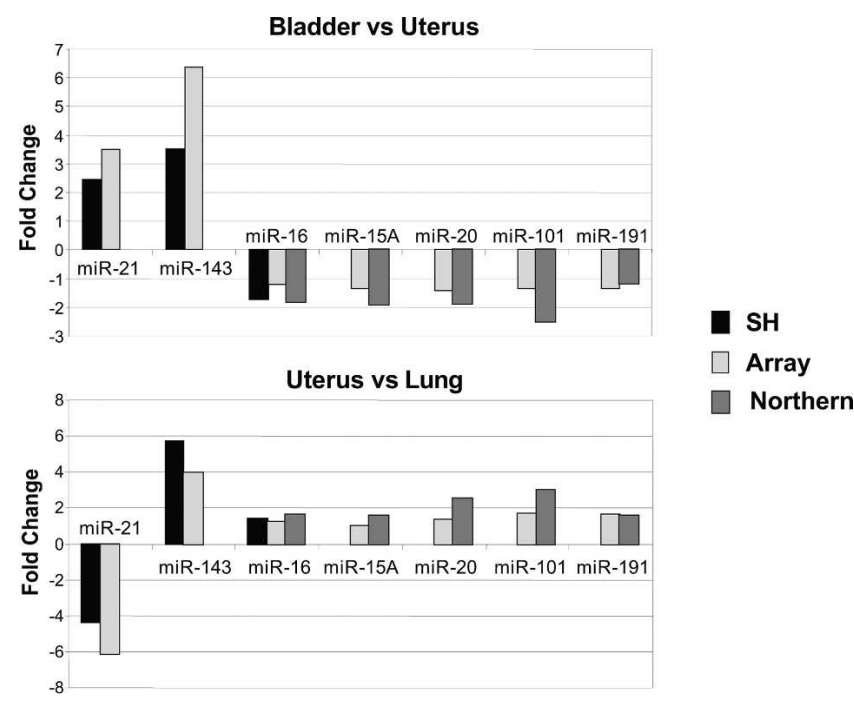

FIGURE 4. Comparison between miRNA microarray data and other detection methods. Total RNA was isolated from human bladder, lung, or uterus. miRNA populations in $10 \mu \mathrm{g}$ of each sample were independently fractionated, labeled, and compared by pairs on two separate arrays (162 probes). In parallel, the same total RNA samples were analyzed by solution hybridization (miR-16, -21 , and $-143,1 \mu \mathrm{g}$ total RNA) or Northern blot (miR-15A, $-16,-20,-101$, and $-191,5 \mu \mathrm{g}$ total RNA) using $5^{\prime}$-end-labeled RNA probes. The graph shows the calculated absolute fold changes between bladder and uterus (top graph) or uterus and lung (bottom graph) deduced from microarray data analysis (Array) and Northern blot (Northern) or solution hybridization $(\mathrm{SH})$ after quantitation by PhosphorImager. 
A

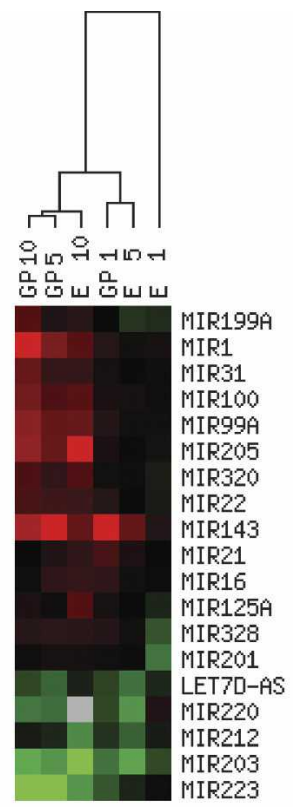

B

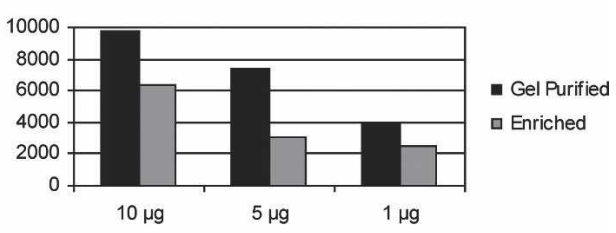

C
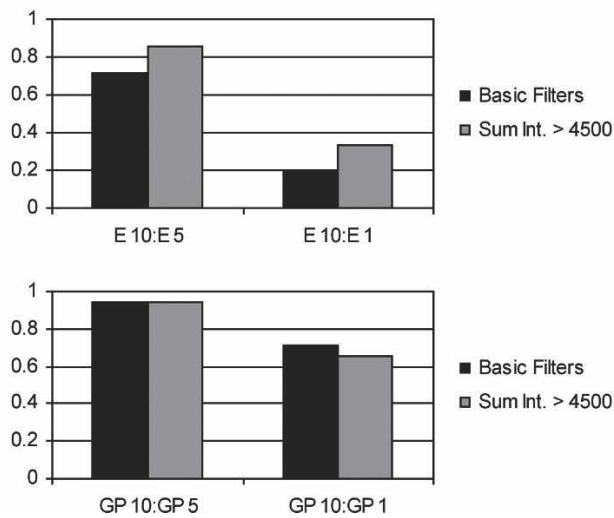

FIGURE 5. Comparison between enriched and gel purified miRNA fractions. One, 5, or $10 \mu \mathrm{g}$ of total RNA from human bladder or lung were used to gel purify miRNAs (GP1, GP5, GP10) or to prepare fractions enriched in small RNA (E1, E5, E10). RNA species in the six lung fractions were labeled with Cy3 and compared with the six Cy5-labeled bladder fractions on six separate arrays (162 probes). (A) miRNA profiles were sorted based on miRNA expression levels across all sample types using hierarchical clustering. Only a few representative examples are shown. Red illustrates higher expression in bladder, green depicts higher expression in lung, and gray represents microarray data that did not pass quality filters (see Materials and Methods). (B) The sum of Cy3 and Cy5 signal intensities for each spot that passed quality filters on the six independent arrays was averaged and plotted for each sample type. The overall intensity signal for the enriched samples was 35\%-60\% lower than for the gel-purified samples. (C) Correlation of miRNA expression levels, i.e., $\log _{2}$ (normalized ratio bladder/uterus signal), in fractions prepared from 5 or $1 \mu \mathrm{g}$ of total RNA relative to the $10 \mu \mathrm{g}$ samples, were calculated for all the spots that passed quality filters (Basic Filters) or only for the spots that had a sum of Cy3 and Cy5 signal intensities superior to 4500 quanta (Sum Int. > 4500). The correlation between enriched fractions (top panel) was better for the high-intensity spots, showing that loss of sensitivity strongly accounts for the loss of accuracy observed with the E5 and E1 fractions.

Most existing array-based miRNA profiling technologies use total RNA or fractions enriched in small RNA by microfiltration or reversed-phase matrices. These procedures then rely on random priming, PCR, or ligation for a labeling step prior to hybridization to immobilized probes. A key improvement in our system was to develop a rapid electrophoretic gel fractionation method that removes the large excess of unrelated RNA species and precursor miRNA sequences that can interfere with the array performance. This purification step corresponds to an enrichment by $\sim 10,000$-fold without affecting the relative miRNA abundance in individual samples (Fig. 4). The resulting fraction is ideal for efficient array labeling as well as other applications, such as cloning or enzymatic amplification (data not shown). A second improvement was to develop a method for homogenous labeling of the miRNA fraction with the highest specific activity without jeopardizing its capacity to hybridize to specific probes on a planar array. Direct enzymatic tailing with PAP and amine-modified NTP results in a homogenous tailed miRNA population that can subsequently be labeled either directly with any amine-reactive molecules, e.g., $\mathrm{Cy}$ or Alexa dyes, or indirectly using NHSbiotin and streptavidin coupled to fluorescent moieties (data not shown). Further, the labeled miRNA fraction is compatible with microarrays created using different immobilization chemistries, such as aldehyde, epoxy, poly- $L$ lysine or three-dimensional matrices glass slides (data not shown). Northern blot, solution hybridization, and microarray experiments clearly demonstrate that the combination of these two improvements does not introduce any bias in the profiling analysis, faithfully conserves miRNA representation, and allows accurate profiling at lower sample input (Figs. 4, 5, 7).

The miRNA microarray system was used to analyze a variety of different human adult tissues. We found that miRNA expression varies dramatically between tissues. Differential miRNA expression across tissues was also confirmed by direct comparison between two tissues and by independent detection methods (Figs. 3B, 4, 7). In contrast, miRNA profiles of identical tissues from multiple donors are very similar (data not shown). Furthermore, most miRNA genes likely to be processed from the same polycistronic primary transcripts have highly correlated expression patterns. As previously reported (Baskerville and Bartel 2005), correlation coefficients for pairs of miRNA genes such as miR-24 and -27a, miR-15a and -16 , or miR-1 and -133 are between 0.74 and 0.91 , indicating that miRNA expression is tightly regulated within individual tissues.

The miRNA expression profiles from different tissues revealed more than 50 miRNAs that are expressed primarily in one or a few related tissues (see Supplemental Table 1). Among these tissue-specific miRNAs was a set that had been previously reported in mouse and/or human: miR-1 in heart and muscle, miR-9, $-124,-125$, and -128 in brain, miR-122A in liver, miR-133A and -206 in muscle, miR-142 and -150 in spleen, or miR-148 in liver and stomach (LagosQuintana et al. 2002; Krichevsky et al. 2003; Babak et al. 2004; Sempere et al. 2004; Baskerville and Bartel 2005). In addition, miRNA expression profiles in several of the tissues assayed here had not been previously reported. The depth of our study (162 miRNAs, 26 tissues) allowed us to complete the human miRNA expression map and to identify new miRNAs with unexpected expression patterns. For 

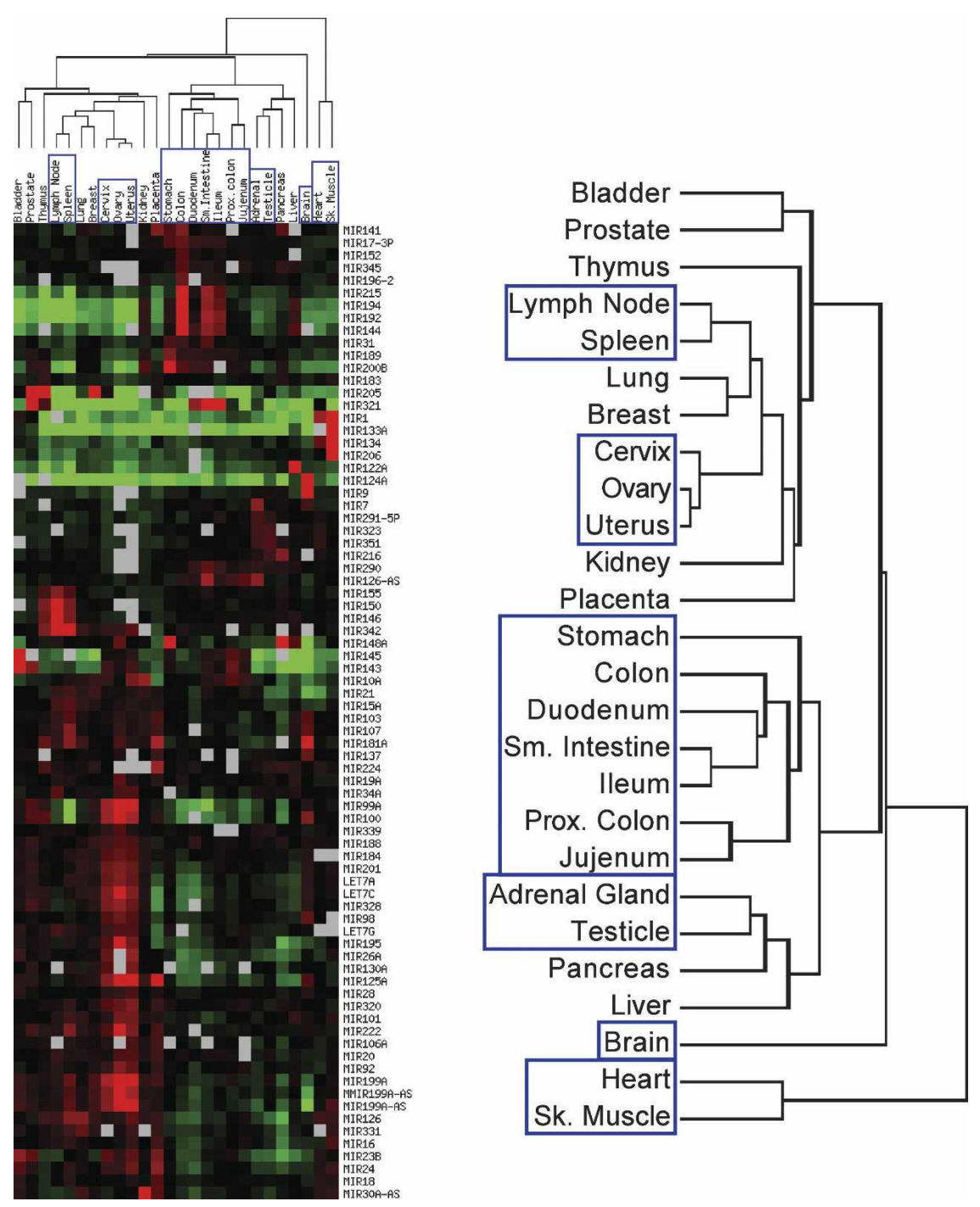

FIGURE 6. miRNA expression profiles across 26 human normal tissues. miRNAs from $10 \mu \mathrm{g}$ of the indicated total RNA samples were gel purified, labeled with Cy5, and compared on 26 independent microarrays against Cy3-labeled miRNAs isolated from $10 \mu \mathrm{g}$ of the same reference pool sample. Hierarchical clustering of the tissues based on their respective miRNA profiles was performed by Average Linkage using uncentered Pearson correlation and $\log _{2}$ (normalized ratio tissue/pool signal). Only miRNAs with a differential expression greater than twofold ( $\mid$ LogRatio $\mid>1$ ) relative to the pool in at least one tissue are shown (78 out of 162 miRNAs, see Supplemental Table 1 at http://www.ambion.com/techlib/data/RNA05b.pdf for all miRNAs). Red illustrates higher expression in a given tissue, green corresponds to higher expression in the pool, and gray represents microarray data that did not pass quality filters (see Materials and Methods). A magnification of the dendrogram is presented on the right. Brain and tissues with miRNA profiles clustering together are boxed.

example, we found that miR-125 is in fact more expressed in cervix, ovary, and uterus than in brain, miR-142 and -150 are not only expressed in spleen but are also very abundant in lymph node, ovary or, thymus, and miR-148 was detected in pancreas and ovary in addition to liver and stomach. miR$181 \mathrm{~A}$ was found highly expressed in brain, placenta, spleen, and thymus, while miR-205 was identified in breast, prostate, and thymus. About 20 miRNAs, such as miR-126-AS, -143, $-145,-189$, or -321 , were found highly expressed in one or several tissues from the digestive tract cluster. Strikingly, the tissues that showed the highest restricted enrichment in individual miRNAs are brain and the cervix/uterus/ovary cluster. This observation suggests that specific miRNAs might play a critical role in brain and female reproductive system development and/or homeostasis.

Hierarchical clustering of the tissues based on their respective miRNA profiles showed that tissues are characterized by unique miRNA expression profiles. Together with the tissue specificity of several miRNAs, this observation is consistent with the concept that miRNAs are important components of tissue development, differentiation, and maintenance of differentiation in adults. Interestingly, related tissues had similar miRNA profiles, as would be predicted for global regulators of tissue differentiation. One tantalizing hypothesis is that miRNAs might directly regulate the expression levels of many of the genes that distinguish these different adult tissues or clusters of adult tissues.

Categorizing miRNAs based on their coregulation or identifying miRNAs that are differentially expressed between different tissues or cell samples will undoubtedly identify interesting miRNAs that deserve closer scrutiny. Further questions are whether these miRNA signatures are affected in disease states and, if so, at what stage. A direct comparison between a large panel of human tumors and their corresponding normal adjacent tissues revealed several miRNAs that might directly contribute to oncogenesis (K. Keiger, J. Shelton, D. Brown, E. Labourier, in prep.). This approach, combined with genetic studies in $C$. elegans and functional studies in human cultured cells, recently helped identify let-7 miRNA as a regulator of RAS oncogene expression and as a potential tumor suppressor disrupted in lung tumors (Johnson et al. 2005). Further, our microarray technology enables accurate miRNA expression profiling in fixed tissues (P. Powers, R. Conrad, K. Keiger, J. Shelton, D. Brown, E. Labourier, in prep.), providing access to a vast amount of information from archived clinical samples. If miRNAs are indeed involved in oncogenesis, inflammatory response, or viral infection, comparative miRNA expression studies in disease samples will certainly reveal novel therapeutic targets and diagnostic markers. 


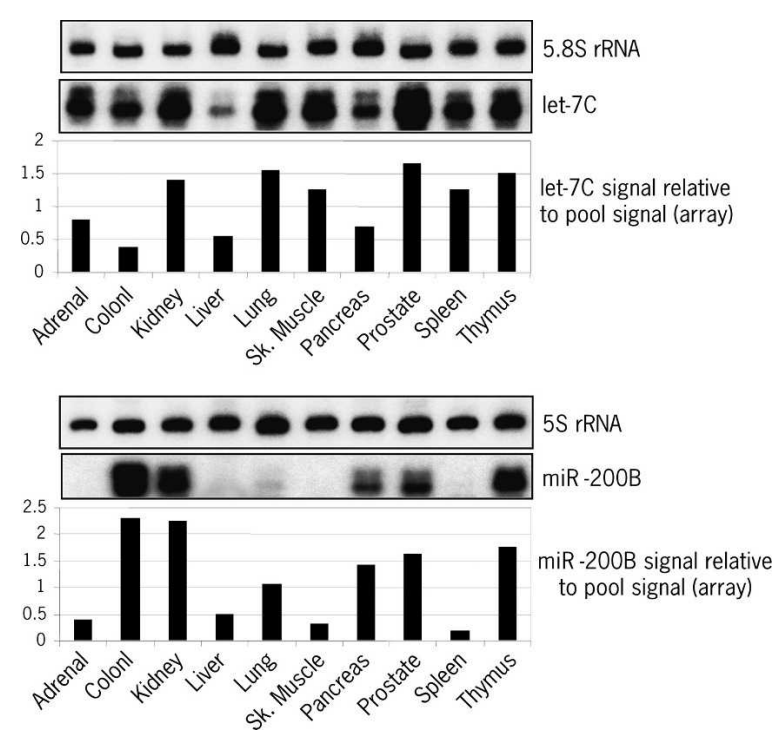

FIGURE 7. Validation of miRNA expression data. Total RNA was isolated from the 10 indicated tissues, independent from the ones used in the microarray profiling study (Fig. 6). Expression levels of let-7C and miR200B were analyzed by Northern blot ( $2 \mu \mathrm{g}$ total RNA) or solution hybridization (1 $\mu \mathrm{g}$ total RNA). As a loading control, $5 \mathrm{~S}$ and $5.8 \mathrm{~S}$ ribosomal RNAs were also analyzed by Northern blot. The graphs under each autoradiograph show miRNA expression levels relative to the pool signal according to the microarray data (see Supplemental Table 1 at http://www.ambion.com/techlib/data/RNA05b.pdf). The value 1 corresponds to the average expression level of the corresponding miRNA in the pool sample, i.e., $\log _{2}($ normalized ratio tissue/pool signal $)=0$.

\section{MATERIALS AND METHODS}

\section{Microarray platform}

DNA oligonucleotide probes from the mirVana miRNA Probe Set (Ambion) were used to print custom microarrays. Each probe was designed using the sequence of its respective mature miRNA according to the miRNA Registry (Griffiths-Jones 2004) and was analyzed for potential secondary structure and cross hybridization. Negative control probes were designed using sequences from Escherichia coli, and each was functionally validated to have minimal cross-hybridization to a collection of chemically synthesized miRNAs as well as a series of tissue-derived miRNAs. Each probe carries a 15-nt linker at the $3^{\prime}$ end of the miRNA complement sequence in addition to an amine group used to couple the probes to coated glass slides. Probes were resuspended in $3 \times$ SSC at 50 $\mu \mathrm{M}$ and spotted on Nexterion Slide E (Schott) at 55\% humidity with the ArrayIt SpotBot (Telechem). Each feature was spotted using SMP4 Stealth pins with a spot diameter of $\sim 100 \mu \mathrm{m}$. The slides were rehydrated by incubation at room temperature in a humidity chamber for $30 \mathrm{~min}$ followed by a 30 -min incubation at $60^{\circ} \mathrm{C}$ to dry. The slides were blocked in a solution containing 100 $\mathrm{mM}$ ethanolamine, $1 \mathrm{M}$ Tris ( $\mathrm{pH} 9.0$ ), and $0.1 \%$ SDS for $15 \mathrm{~min}$ at $50^{\circ} \mathrm{C}$, then thoroughly rinsed with water and spun dry.

\section{RNA preparation}

Total RNA isolation and small RNA enrichment procedure were performed with the mirVana miRNA Isolation Kit (Ambion) according to the manufacturer's instructions. For miRNA expression profiling in normal human tissues, miRNA certified FirstChoice Total RNA (Ambion) were used. To isolate miRNA fractions, total RNA samples were fractionated and cleaned up with the flashPAGE Fractionator and reagents (Ambion) per the manufacturer's recommendation. Briefly, 1-20 $\mu$ g of each RNA sample were loaded onto the top of a column filled with a denaturing acrylamide gel matrix and fractionated by applying an electrical current. A dye was loaded with the total RNA sample to track RNAs that are $\sim 40 \mathrm{nt}$ in size. Electrophoresis was stopped when the dye reached the bottom of the column, and miRNAs were recovered from the bottom buffer chamber using a glass fiber filter-based cleaning procedure (flashPAGE Reaction Cleanup Kit, Ambion). Approximately $1 \mathrm{ng}$ of miRNA was recovered per $10 \mu \mathrm{g}$ of total RNA.

\section{miRNA labeling and cleanup}

Chemically synthesized oligoribonucleotides (Ambion), purified miRNAs, or fractions enriched in small RNAs were labeled with the mirVana miRNA Labeling Kit (Ambion) and amine-reactive dyes as recommended by the manufacturer. Poly(A) polymerase and a mixture of unmodified and amine-modified nucleotides were first used to append a poly-nucleotide tail to the $3^{\prime}$ end of each miRNA. The amine-modified miRNAs were then cleaned up and coupled to NHS-ester modified Cy5 or Cy3 dyes (Amersham Bioscience). Unincorporated dyes were removed with a second glass fiber filter-based cleaning procedure.

\section{Microarray hybridization and data analysis}

A $3 \times$ miRNA Hybridization Buffer (Ambion) was added to the fluorescently labeled miRNAs and the solution was heated at $95^{\circ} \mathrm{C}$ for $3 \mathrm{~min}$. Slides were hybridized $12-16 \mathrm{~h}$ at $42^{\circ} \mathrm{C}$ in sealed cassettes using a water bath. Following hybridization, the slides were washed and dried prior to a high-resolution scan on a GenePix 4000B Array Scanner (Axon). Each element was located and analyzed using the GenePix Pro 5.0 software package (Axon). Data were filtered for quality and significance using the Longhorn Array Database (Killion et al. 2003). Filters were based on several data quality standards, including minimum intensity and pixel consistency. All data used for analysis had a signal-to-noise ratio $>5$, an average sum intensity $50 \%$ higher than that of the negative control spots, and a regression ratio $>0.5$. Data were normalized globally per array such that the average LogRatio was 0 after normalization. Hierarchical clustering was performed by Average Linkage using uncentered Pearson correlation (Eisen et al. 1998).

\section{Microarray data validation}

miRNA expression levels in total RNA samples were measured by Northern blot $(2-5 \mu \mathrm{g})$ or solution hybridization $(1 \mu \mathrm{g})$ with the mirVana miRNA Detection Kit (Ambion) according to the manufacturer's instructions. DNA or RNA probes were labeled and purified with the mirVana Probe \& Marker Kit (Ambion) and $\left[\gamma-{ }^{32} \mathrm{P}\right] \mathrm{ATP}$ at $6000 \mathrm{Ci} / \mathrm{mmol}$ (PerkinElmer). For Northern analyses, RNA samples were resolved on denaturing $15 \%$ polyacrylamide gels and electroblotted on BrightStar-Plus Nylon membranes (Ambion). 
Membranes were blocked in UltraHyb-oligo Hybridization Buffer (Ambion) for at least $1 \mathrm{~h}$ at $65^{\circ} \mathrm{C}$, hybridized overnight at $42^{\circ} \mathrm{C}$ with the appropriate probe, and washed three times with NorthernMax Low Stringency Wash Buffer (Ambion) for $5 \mathrm{~min}$ at room temperature followed by $15 \mathrm{~min}$ at $42^{\circ} \mathrm{C}$. Results were quantified on a PhosphorImager Storm 860 (Amersham Bioscience).

\section{SUPPLEMENTARY MATERIAL}

Supplementary material can be found at http://www.ambion. com/techlib/data/RNA05b.pdf.

\section{AKNOWLEDGMENTS}

We thank Eric Devroe for helpful comments on this article. This work was supported in part by National Genome Research Institute SBIR grant R43HG003430 to D.B.

Received April 6, 2005; accepted June 3, 2005.

\section{REFERENCES}

Allawi, H.T., Dahlberg, J.E., Olson, S., Lund, E., Olson, M., Ma, W.P., Takova, T., Neri, B.P., and Lyamichev, V.I. 2004. Quantitation of microRNAs using a modified Invader assay. RNA 10: 1153-1161.

Ambros, V. 2004. The functions of animal microRNAs. Nature 431: 350-355.

Babak, T., Zhang, W., Morris, Q., Blencowe, B.J., and Hughes, T.R. 2004. Probing microRNAs with microarrays: Tissue specificity and functional inference. RNA 10: 1813-1819.

Barad, O., Meiri, E., Avniel, A., Aharonov, R., Barzilai, A., Bentwich, I., Einav, U., Gilad, S., Hurban, P., Karov, Y., et al. 2004. MicroRNA expression detected by oligonucleotide microarrays: System establishment and expression profiling in human tissues. Genome Res. 14: 2486-2494.

Bartel, D.P. and Chen, C.Z. 2004. Micromanagers of gene expression: The potentially widespread influence of metazoan microRNAs. Nat. Rev. Genet. 5: 396-400.

Baskerville, S. and Bartel, D.P. 2005. Microarray profiling of microRNAs reveals frequent coexpression with neighboring miRNAs and host genes. RNA 11: 241-247.

Bohnsack, M.T., Czaplinski, K., and Gorlich, D. 2004. Exportin 5 is a RanGTP-dependent dsRNA-binding protein that mediates nuclear export of pre-miRNAs. RNA 10: 185-191.

Cai, X., Hagedorn, C.H., and Cullen, B.R. 2004. Human microRNAs are processed from capped, polyadenylated transcripts that can also function as mRNAs. RNA 10: 1957-1966.

Denli, A.M., Tops, B.B., Plasterk, R.H., Ketting, R.F., and Hannon, G.J. 2004. Processing of primary microRNAs by the Microprocessor complex. Nature 432: 231-235.

Doench, J.G., Petersen, C.P., and Sharp, P.A. 2003. siRNAs can function as miRNAs. Genes \& Dev. 17: 438-442.

Eisen, M.B., Spellman, P.T., Brown, P.O., and Botstein, D. 1998. Cluster analysis and display of genome-wide expression patterns. Proc. Natl. Acad. Sci. 95: 14863-14868.

Grad, Y., Aach, J., Hayes, G.D., Reinhart, B.J., Church, G.M., Ruvkun, G., and Kim, J. 2003. Computational and experimental identification of C. elegans microRNAs. Mol. Cell 11: 1253-1263.

Gregory, R.I., Yan, K.P., Amuthan, G., Chendrimada, T., Doratotaj, B., Cooch, N., and Shiekhattar, R. 2004. The Microprocessor complex mediates the genesis of microRNAs. Nature 432: 235-240.

Griffiths-Jones, S. 2004. The microRNA registry. Nucleic Acids Res. 32: D109-111.
Grishok, A., Pasquinelli, A.E., Conte, D., Li, N., Parrish, S., Ha, I., Baillie, D.L., Fire, A., Ruvkun, G., and Mello, C.C. 2001. Genes and mechanisms related to RNA interference regulate expression of the small temporal RNAs that control C. elegans developmental timing. Cell 106: 23-34.

Hartig, J.S., Grune, I., Najafi-Shoushtari, S.H., and Famulok, M. 2004. Sequence-specific detection of MicroRNAs by signal-amplifying ribozymes. J. Am. Chem. Soc. 126: 722-723.

Houbaviy, H.B., Murray, M.F., and Sharp, P.A. 2003. Embryonic stem cell-specific microRNAs. Dev. Cell 5: 351-358.

Hutvagner, G., McLachlan, J., Pasquinelli, A.E., Balint, E., Tuschl, T., and Zamore, P.D. 2001. A cellular function for the RNA-interference enzyme Dicer in the maturation of the let-7 small temporal RNA. Science 293: 834-838.

Johnson, S.M., Grosshans, H., Shingara, J., Byrom, M., Jarvis, R., Cheng, A., Labourier, E., Reinert, K.L., Brown, D., and Slack, F.J. 2005. RAS is regulated by the let-7 microRNA family. Cell 120: 635-647.

Ketting, R.F., Fischer, S.E., Bernstein, E., Sijen, T., Hannon, G.J., and Plasterk, R.H. 2001. Dicer functions in RNA interference and in synthesis of small RNA involved in developmental timing in $C$. elegans. Genes \& Dev. 15: 2654-2659.

Killion, P.J., Sherlock, G., and Iyer, V.R. 2003. The Longhorn Array Database (LAD): An open-source, MIAME compliant implementation of the Stanford Microarray Database (SMD). BMC Bioinform. 4: 32.

Krichevsky, A.M., King, K.S., Donahue, C.P., Khrapko, K., and Kosik, K.S. 2003. A microRNA array reveals extensive regulation of microRNAs during brain development. RNA 9: 1274-1281.

Lagos-Quintana, M., Rauhut, R., Yalcin, A., Meyer, J., Lendeckel, W., and Tuschl, T. 2002. Identification of tissue-specific microRNAs from mouse. Curr. Biol. 12: 735-739.

Lee, Y., Jeon, K., Lee, J.T., Kim, S., and Kim, V.N. 2002. MicroRNA maturation: Stepwise processing and subcellular localization. EMBO J. 21: 4663-4670.

Lee, Y., Ahn, C., Han, J., Choi, H., Kim, J., Yim, J., Lee, J., Provost, P., Radmark, O., Kim, S., et al. 2003. The nuclear RNase III Drosha initiates microRNA processing. Nature 425: 415-419.

Lee, Y., Kim, M., Han, J., Yeom, K.H., Lee, S., Baek, S.H., and Kim, V.N. 2004. MicroRNA genes are transcribed by RNA polymerase II. EMBO J. 23: 4051-4060.

Lim, L.P., Lau, N.C., Weinstein, E.G., Abdelhakim, A., Yekta, S., Rhoades, M.W., Burge, C.B., and Bartel, D.P. 2003. The microRNAs of Caenorhabditis elegans. Genes \& Dev. 17: 991-1008.

Liu, C.G., Calin, G.A., Meloon, B., Gamliel, N., Sevignani, C., Ferracin, M., Dumitru, C.D., Shimizu, M., Zupo, S., Dono, M., et al. 2004. An oligonucleotide microchip for genome-wide microRNA profiling in human and mouse tissues. Proc. Natl. Acad. Sci. 101: 9740-9744.

Llave, C., Xie, Z., Kasschau, K.D., and Carrington, J.C. 2002. Cleavage of Scarecrow-like mRNA targets directed by a class of Arabidopsis miRNA. Science 297: 2053-2056.

Lund, E., Guttinger, S., Calado, A., Dahlberg, J.E., and Kutay, U. 2004. Nuclear export of microRNA precursors. Science 303: 95-98.

Miska, E.A., Alvarez-Saavedra, E., Townsend, M., Yoshii, A., Sestan, N., Rakic, P., Constantine-Paton, M., and Horvitz, H.R. 2004. Microarray analysis of microRNA expression in the developing mammalian brain. Genome Biol. 5: R68.

Murchison, E.P., and Hannon, G.J. 2004. miRNAs on the move: miRNA biogenesis and the RNAi machinery. Curr. Opin. Cell Biol. 16: 223-229.

Nelson, P.T., Baldwin, D.A., Scearce, L.M., Oberholtzer, J.C., Tobias, J.W., and Mourelatos, Z. 2004. Microarray-based, high-throughput gene expression profiling of microRNAs. Nat. Methods 1: 155-161.

Olsen, P.H. and Ambros, V. 1999. The lin-4 regulatory RNA controls developmental timing in Caenorhabditis elegans by blocking LIN14 protein synthesis after the initiation of translation. Dev. Biol. 216: 671-680.

Overhoff, M., Wunsche, W., and Sczakiel, G. 2004. Quantitative detection of siRNA and single-stranded oligonucleotides: Relationship between uptake and biological activity of siRNA. Nucleic Acids Res. 32: e170. 
Pfeffer, S., Zavolan, M., Grasser, F.A., Chien, M., Russo, J.J., Ju, J., John, B., Enright, A.J., Marks, D., Sander, C., et al. 2004. Identification of virus-encoded microRNAs. Science 304: 734736.

Rodriguez, A., Griffiths-Jones, S., Ashurst, J.L., and Bradley, A. 2004. Identification of mammalian microRNA host genes and transcription units. Genome Res. 14: 1902-1910.

Schmittgen, T.D., Jiang, J., Liu, Q., and Yang, L. 2004. A highthroughput method to monitor the expression of microRNA precursors. Nucleic Acids Res. 32: e43.

Schwarz, D.S., Hutvagner, G., Du, T., Xu, Z., Aronin, N., and Zamore, P.D. 2003. Asymmetry in the assembly of the RNAi enzyme complex. Cell 115: 199-208.

Sempere, L.F., Freemantle, S., Pitha-Rowe, I., Moss, E., Dmitrovsky, E., and Ambros, V. 2004. Expression profiling of mammalian microRNAs uncovers a subset of brain-expressed microRNAs with possible roles in murine and human neuronal differentiation. Genome Biol. 5: R13.

Sioud, M. and Rosok, O. 2004. Profiling microRNA expression using sensitive cDNA probes and filter arrays. Biotechniques 37: 574-576, $578-580$.

Sun, Y., Koo, S., White, N., Peralta, E., Esau, C., Dean, N.M., and Perera, R.J. 2004. Development of a micro-array to detect human and mouse microRNAs and characterization of expression in human organs. Nucleic Acids Res. 32: e188.
Thomson, J.M., Parker, J., Perou, C.M., and Hammond, S.M. 2004. A custom microarray platform for analysis of microRNA gene expression. Nat. Methods 1: 47-53.

Valoczi, A., Hornyik, C., Varga, N., Burgyan, J., Kauppinen, S., and Havelda, Z. 2004. Sensitive and specific detection of microRNAs by Northern blot analysis using LNA-modified oligonucleotide probes. Nucleic Acids Res. 32: e175.

Yekta, S., Shih, I.H., and Bartel, D.P. 2004. MicroRNA-directed cleavage of HOXB8 mRNA. Science 304: 594-596.

Yi, R., Qin, Y., Macara, I.G., and Cullen, B.R. 2003. Exportin-5 mediates the nuclear export of pre-microRNAs and short hairpin RNAs. Genes \& Dev. 17: 3011-3016.

Zeng, Y. and Cullen, B.R. 2003. Sequence requirements for micro RNA processing and function in human cells. RNA 9: 112-123.

. 2004. Structural requirements for pre-microRNA binding and nuclear export by Exportin 5. Nucleic Acids Res. 32: 4776-4785.

Zeng, Y., Wagner, E.J., and Cullen, B.R. 2002. Both natural and designed micro RNAs can inhibit the expression of cognate mRNAs when expressed in human cells. Mol. Cell 9: 1327-1333.

Zeng, Y., Yi, R., and Cullen, B.R. 2005. Recognition and cleavage of primary microRNA precursors by the nuclear processing enzyme Drosha. EMBO J. 24: 138-148.

Zhang, H., Kolb, F.A., Jaskiewicz, L., Westhof, E., and Filipowicz, W. 2004. Single processing center models for human Dicer and bacterial RNase III. Cell 118: 57-68. 

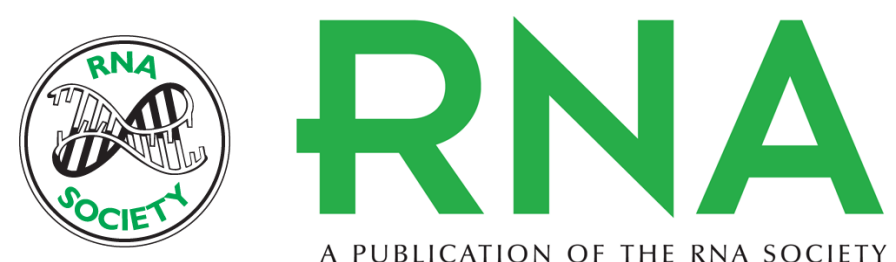

A PUBLICATION OF THE RNA SOCIETY

\section{An optimized isolation and labeling platform for accurate microRNA expression profiling}

JACLYN SHINGARA, KERRI KEIGER, JEFFREY SHELTON, et al.

RNA 2005 11: 1461-1470

References This article cites 51 articles, 22 of which can be accessed free at:

http://rnajournal.cshlp.org/content/11/9/1461.full.html\#ref-list-1

\section{License}

Email Alerting Receive free email alerts when new articles cite this article - sign up in the box at the Service top right corner of the article or click here. 